\title{
Coronal Magnetic Flux Ropes in Quadrupolar Magnetic Fields
}

\author{
Yingzhi Zhang ${ }^{1}$, Youqiu $\mathbf{H u}^{2}$ \\ and Jingxiu Wang ${ }^{1}$
}

1.National Astronomical Observatories, Chinese Academy of Sciences, Beijing 100012, China email:zhangyingzhi@ourstar.bao.ac.cn

2.School of Earth and Space Sciences, University of Science and Technology of China, Hefei 230026, China

\begin{abstract}
Using a 2.5-D, time-dependent ideal MHD model in spherical coordinates, we carry out a numerical study of the equilibrium properties of coronal magnetic flux ropes in a quadrupolar background magnetic field. For such a flux rope system, a catastrophic occurs: the flux rope is detached from the photosphere and jumps to a finite altitude with a vertical current sheet below. There is a transversal current sheet formed above the rope, and the whole system stays in quasi-equilibrium. We argue that the additional Lorentz force provided by the transversal current sheet on the flux rope plays an important role in keeping the system in quasi-equilibrium in the corona.
\end{abstract}

Keywords. Sun: corona, magnetic fields, MHD

\section{Introduction}

A common conclusion of many numerical studies is that catastrophe exists under certain conditions. In spherical geometry, however, catastrophe exists for the flux rope system with a bipolar background field that may be either partly open or completely closed (Hu et al., 2003), and the catastrophe amplitude is infinite.

\section{Numerical Results}

We work out the 2.5-dimensional ideal MHD equations in spherical coordinates $(\mathrm{r}, \theta, \varphi)$. The initial magnetic field is of Antiochos type (Antiochos et al, 1999). The multistep implicit scheme $(\mathrm{Hu}, 1989)$ is used to solve the 2.5-dimensional ideal MHD equations.

In this study, calculations were carried out for different values of the axial magnetic flux $\phi_{z}$ for a fixed annular magnetic flux $\phi_{p}=0.5$. Figure 1 shows the height of the rope axis $h_{a}$ and the length of the vertical current sheet $h_{c}$ as a function of $\phi_{z}$ for the quasi-equilibrium states thus obtained. As seen from this figure, for $\phi_{z} \leqslant 0.0437$, the flux rope remains attached to the solar surface but expands with increasing $\phi_{z}$. As a result, $h_{c}$ is zero and $h_{a}$ increases slightly with increasing $\phi_{z}$. For $\phi_{z} \approx 0.0438$, the two geometric parameters jump to about 1.7 and 2.6, respectively, implying a catastrophe of finite amplitude. We show the magnetic field lines at several separate times in Figure 2 for $\phi_{z}=0.0437$ (Figure 2(A-E)) and $\phi_{z}=0.0438$ (Figure 2(F-J)). For both cases, the original neutral point changes to a transversal current sheet under the action of the flux rope, and it provides a downward Lorentz force on the rope. After a temporal evolution of 150 $\tau_{A}$ (in Figure $2 \tau$ represents $\tau_{A}$ ), the system as a whole approaches quasi-equilibrium for both cases. The magnetic rope sticks to the photosphere for $\phi_{z}=0.0437$, and breaks away from the photosphere, leaving a vertical current sheet stretching from the photosphere 


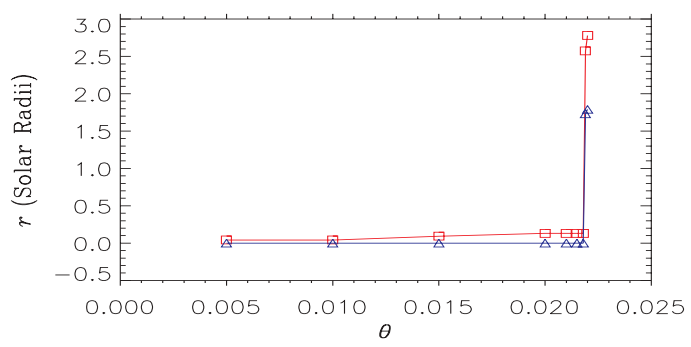

Figure 1. Geometrical properties of the coronal magnetic flux rope in equilibrium versus the axial magnetic flux $\phi_{z}$. The solid squares and the triangles are symbols of $h_{a}$ and $h_{c}$.
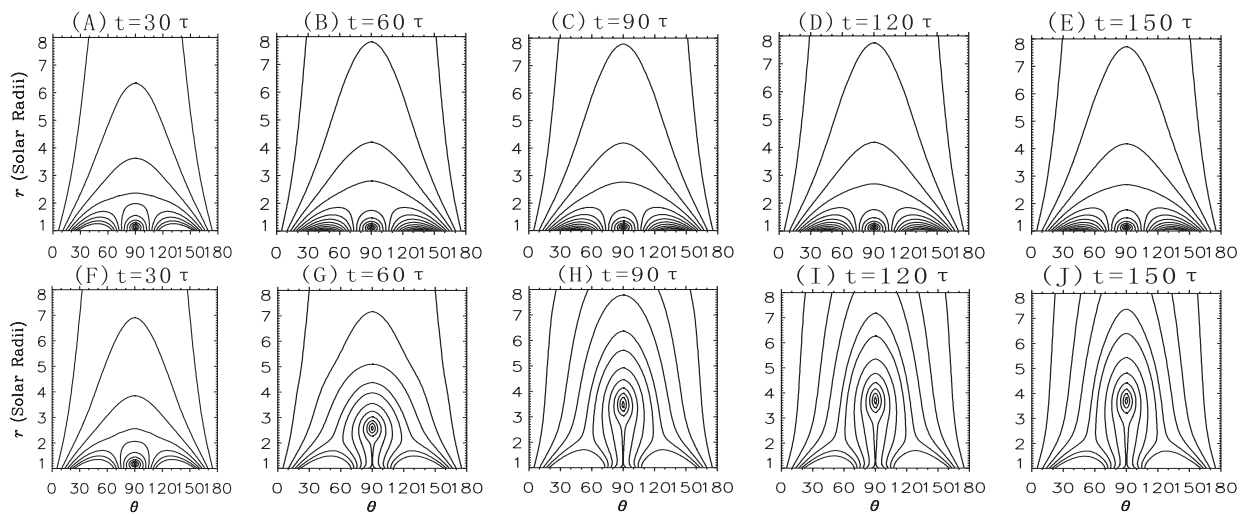

Figure 2. Magnetic field lines at several times for $\phi_{z}=0.0437(\mathrm{~A}-\mathrm{E})$ and $\phi_{z}=0.0438(\mathrm{~F}-\mathrm{J})$

to the bottom of the rope for $\phi_{z}=0.0438$. Besides, the flux rope stands in the corona for a long time in the second cases, as seen from Figure 2(F-J).

\section{Concluding Remarks}

This study shows that the system exhibits a catastrophic behavior in response to the enhancement of the axial magnetic fluxes of the rope, but the catastrophic amplitude is finite. The flux rope may levitate stably in the corona instead of escaping to infinity. This forms a striking contrast to the bipolar background field case as discussed by $\mathrm{Hu}$ et al. (2003). A transversal current sheet forms above the flux rope because of the presence of the neutral point and the subsequent action of the expanding flux rope.We argue that the sheet provides a downward Lorentz force on the flux rope and thus has made the flux rope levitate stably in the corona and has made the associated catastrophe be finite in amplitude.

\section{Acknowledgements}

The work is supported by the National Natural Science Foundation of China(10233050) and the National Key Basic Science Foundation(TG2000078404).

\section{References}

Antiochos, S.K., Devore, C.R. \& Klimchuk, J.A. 1999, ApJ 510, 485

Hu, Y.Q. 1989, J. Comput. Phys. 84, 441

Hu, Y.Q., Li, G.Q. \& Xing, X.Y. 2003, J. Geophys. Res. 108, 1072, DOI: 10.1029/2002JA009419 